\title{
A CHARACTERIZATION OF THE INVARIANT SUBSPACES OF DIRECT SUMS OF STRICTLY CYCLIC ALGEBRAS
}

\author{
ERIK ROSENTHAL
}

\begin{abstract}
Two characterizations of the invariant subspace lattice of $A^{(n)}$ for a strictly cyclic operator algebra $A$ on a separable Hilbert space are proven.
\end{abstract}

We derive a characterization of the invariant subspaces of $A^{(n)}$, where $A$ is an Abelian, hereditarily strictly cyclic operator algebra on a separable Hilbert space. A further characterization is derived in the case when $A$ is generated by a single operator. The first result is also generalized to $A^{(\infty)}$ when $A$ has Donoghue lattice.

We will need some notation and definitions. Throughout this paper, $H$ will be a separable, infinite-dimensional, complex Hilbert space, and $B(H)$ the algebra of all bounded linear operators on $H$. If $T \in B(H)$, then $A(T)$ is the weakly closed subalgebra of $B(H)$ generated by $T$ and the identity. If $F \subset B(H)$, then Lat $F$ is the lattice of all invariant subspaces of $F$. We call $F$ transitive if Lat $F=\{\{0\}, H\}$, and we call $F$ unicellular if Lat $F$ is totally ordered. By $H^{(n)}$ we mean the direct sum of $n$ copies of $H$. For $T \in B(H), T^{(n)}$ is the operator on $H^{(n)}$ defined by

$$
T^{(n)}\left(x_{1}, x_{2}, \ldots, x_{n}\right)=\left(T x_{1}, T x_{2}, \ldots, T x_{n}\right),
$$

and $F^{(n)}=\left\{T^{(n)}: T \in F\right\}$. If $M$ is a subspace of $H^{(n)}$, the $i$ th kernel of $M$ is the collection of all vectors in $M$ whose $i$ th coordinate is 0 . Note that if $M \in \operatorname{Lat} T^{(n)}$, then the $i$ th kernel of $M$ is invariant under $T^{(n)}$, and it is obviously isomorphic to an element of Lat $T^{(n-1)}$. If $F^{(n)} \subset B\left(H^{(n)}\right)$, and if $M \in$ Lat $F^{(n)}$, then $M$ is an invariant graph subspace of $F^{(n)}$ on the ith coordinate if $M$ has the form

$$
M=\left\{\left(T_{1} x, T_{2} x, \ldots, T_{\lambda-1} x, x, T_{i+1} x, \ldots, T_{n} x\right): x \in D\right\}
$$

for some linear manifold $D$ of $H$ and for linear transformations $T_{i}$ with domain $D$ and range contained in $H$. The $T_{i}$ 's will be called graph transformations for $F$. In general, the $T_{i}$ 's need not be closed, although jointly they are closed since $M$ is closed. If $M$ and $N$ are subspaces of $H$, we use $M \vee N$ for the (closed linear) span of $M$ and $N$.

Note that if $M$ is an invariant subspace of $F^{(n)}$, then $M$ is a graph subspace on the $i$ th coordinate if and only if the $i$ th kernel is $\{0\}$; i.e., if and only if the $i$ th coordinate of a vector determines the vector. Note also that the domain of a graph transformation for $F$ is invariant under $F$, and the transformation commutes with

Received by the editors March 14, 1983 and, in revised form, October 19, 1983.

1980 Mathematics Subject Classification. Primary 47A15, 47B99, 47D25; Secondary 15 A04.

(c)1985 American Mathematical Society $0002-9939 / 85 \$ 1.00+\$ .25$ per page 
every operator in $F$. In particular, if $T$ is a graph transformation, so is $T-\lambda$ for any scalar $\lambda$.

Recall that a subalgebra $A$ of $B(H)$ has finite strict multiplicity if there is a finite collection of vectors $E=\left\{x_{1}, x_{2}, \ldots, x_{n}\right\}$ such that

$$
\left\{T_{1} x_{1}+T_{2} x_{2}+\cdots+T_{n} x_{n}: T_{i} \in A\right\}=H .
$$

The minimal cardinality of all such sets $E$ is called the strict multiplicity of $A$. If $A$ has strict multiplicity $1, A$ is said to be strictly cyclic. An operator $T$ has finite strict multiplicity if $A(T)$ does, and $T$ is strictly cyclic if $A(T)$ is. An operator algebra $A$ is said to be hereditarily strictly cyclic if the uniform closure of its restriction to every invariant subspace is strictly cyclic. The vector $x$ is a separating vector for $A$ if $T x=0$ implies $T=0$ whenever $T$ is an operator in $A$. Note that cyclic vectors for Abelian operator algebras are always separating.

The following propositions are known results which we will need.

Proposition 1 (HeRRERo [7]). A uniformly closed operator algebra of finite strict multiplicity has no dense invariant manifolds other than $H$.

Proposition 2 (LAMBert [11]). If $T$ is an operator in an Abelian strictly cyclic algebra, then the spectrum of $T$ consists entirely of compression spectrum. (Recall that the compression spectrum of $T$ is the set of complex numbers $\lambda$ such that the range of $T-\lambda$ is not dense in $H$.)

Proposition 3 (HerRero [8]). Every densely defined linear transformation commuting with every operator in an algebra of finite strict multiplicity is bounded.

Proposition 4 (Rosenthal [20]). Let $T$ be strictly cyclic. If $T$ is unicellular, then $T$ has one-point spectrum. Conversely, if $T$ is hereditarily strictly cyclic, and if $T$ has one-point spectrum, then $T$ has Donoghue lattice (i.e., there is an orthonormal basis $\left\{e_{i}\right\}_{i=0}^{\infty}$ of $H$ such that the nontrivial invariant subspaces of $T$ are the subspaces $M_{k}=\bigvee_{i=k}^{\infty} e_{i}$ for positive integers $k$ ).

THEOREM 1. Let $A$ be an Abelian, unicellular, hereditarily strictly cyclic algebra. Then every element of Lat $A^{(n)}$ can be expressed as the span of at most $n$ invariant graph subspaces whose domains are in Lat $A$.

Proof. Let $M \in$ Lat $A^{(n)}$, and let $P_{i}$ be the $i$ th coordinate projection. Since Lat $A$ is totally ordered and each $\overline{P_{i} M}$ (the closure of $P_{i} M$ ) is in Lat $A$, we can choose $i_{0}$ such that $P_{i} M \subset \overline{P_{i_{0}} M}$ for $i=1,2, \ldots, n$.

Claim. $P_{i_{0}} M$ is closed.

Proof of Claim. Let $\tilde{A}$ be the closure of $A / \overline{P_{i_{0}} M}$, and let $N={\overline{P_{i_{0}} M}}^{(n)}$. The algebra $A$ is strictly cyclic by hypothesis, and, obviously, $\tilde{A}^{(n)}$ is the (uniform) closure of $A^{(n)} / N$. In particular, $M \in$ Lat $\tilde{A}^{(n)}$, so $P_{i_{0}} M$ is invariant under $\tilde{A}$. Of course, $P_{i_{0}} M$ is dense in $\overline{P_{i_{0}} M}$, and so by Proposition $1, P_{i_{0}} M=\overline{P_{i_{0}} M}$, and the claim is proven.

The above conclusion implies that we can choose a vector $x_{0} \in P_{i_{0}} M$ which is strictly cyclic for $\tilde{A}$. Choose $f_{0} \in M$ such that $P_{i_{0}} f_{0}=x_{0}$, and let

$$
G_{0}=A^{(n)} f_{0}, \quad G=\tilde{A}^{(n)} f_{0} .
$$


Every vector in $G$ is a limit of vectors in $G_{0}$ since every operator in $\tilde{A}$ is a limit of operators in $A / P_{i_{0}} M$. Hence, $G$ is invariant under $A^{(n)}$. If we show that $G$ is a graph on the $i_{0}$ th coordinate, we will know that $G$ is closed since its graph transformations will be densely defined and commute with every operator in an Abelian strictly cyclic algebra; Proposition 3 then implies that they will be bounded. Since the domain of $G$ is $P_{i_{0}} M$, its domain is in Lat $A$.

To show that $G$ is a graph on the $i$ th coordinate, it is enough to show that if $\left(y_{1}, y_{2}, \ldots, y_{n}\right)$ is in $G$ with $y_{i_{0}}=0$, then $y_{i}=0$ for $i=1,2, \ldots, n$. But this is clear since $x_{0}$ is a separating vector; i.e., if $T x_{0}=y_{i_{0}}=0$, then $T=0$, so $T^{(n)} f_{0}=0$.

Let $K$ be the $i_{0}$ th kernel of $M$. Trivially, $K \in$ Lat $A^{(n)}$; and if $x \in M$, there exists a $y$ in $G$ such that $P_{i_{0}} x=P_{i_{0}} y$ (since $\left.P_{i_{0}} G=P_{i_{0}} M\right)$, so $x-y \in K$. Thus, $y+(x-y)$ $\in M$, so $M=G \vee K$. We can now perform the same procedure on $K$. Since $P_{i_{0}} K=\{0\}$, the index chosen will be different from $i_{0}$, and the next kernel chosen will have at least two coordinate projections which are $\{0\}$. We continue in this manner getting $n$ invariant graph subspaces of $A^{(n)}$. Since the number of zero coordinate projections increases at each step, and since $M$ is obviously the span of these subspaces, we are done.

A little more can be said about the structure of the graphs. If $G_{1}, G_{2}, \ldots, G_{n}$ are the graph subspaces in the order chosen in the proof, then $G_{n}$ is a coordinate subspace; $G_{n-1}$ is 0 on the domain slots of $G_{1}, \ldots, G_{n-2} ; G_{n-2}$ is 0 on the domain slots of $G_{1}, \ldots, G_{n-3}$; and so on. (Of course, some of the $G_{i}$ 's may be 0 .)

Nikolskii [15] has shown that if $T$ is a weighted shift with $p$-summable weights $(p>0)$ which go monotonically to 0 , then $T$ has Donoghue lattice (Nikolskii actually obtains more general conditions for unicellularity). Lambert [11] has shown that such an operator is strictly cyclic. Since the restriction of $T$ to any invariant subspace is another such operator, the algebra generated by $T$ satisfies the hypothesis of the theorem. Nordgren [17] has shown that every Donoghue operator $T$ has the property that each of the invariant subspaces of $\left(T^{*}\right)^{(n)}$ is a span of finite-dimensional invariant subspaces. The following theorem shows that this is true for a much wider class of operators.

THEOREM 2. Let $T$ be hereditarily strictly cyclic with $\sigma(T)=\{\lambda\}$. Then if $M \in$ Lat $\left(T^{*}\right)^{(n)}, M$ is a span of finite-dimensional invariant subspaces of $\left(T^{*}\right)^{(n)}$. Equivalently, if $M \in \mathrm{Lat} T^{(n)}, M$ is an intersection of invariant subspaces of $T^{(n)}$ of finite codimension.

Proof. Replacing $T$ by $T-\lambda$ if necessary, we can assume that $\lambda=0$. By Theorem 1 we need only consider invariant graph subspaces whose domains are subspaces of $H$. So let

$$
G=\left\{\left(x, T_{1} x, T_{2} x, \ldots, T_{n-1} x\right): x \in D\right\}
$$

be a graph subspace of $T^{(n)}$. The subspace $D$ is in Lat $T$ and, by Proposition 4 , has the form $D=\bigvee_{i=k}^{\infty} e_{i}$ for an orthonormal basis $\{e\}_{i=0}^{\infty}$.

Let $M_{j}=\bigvee_{i=j}^{\infty} e_{i}$ (so $D=M_{k}$ ). Then, for each $j, M_{j}^{(n)}$ is invariant under $T^{(n)}$ and has finite codimension. Let

$$
f_{k}=\left(e_{k}, T_{1} e_{k}, \ldots, T_{n-1} e_{k}\right) \text { and } f_{k}^{i}=\left(T^{(n)}\right)^{i} f_{k} .
$$


Then $f_{k} \in G$, and so $f_{k}^{i} \in G$ since $G \in$ Lat $T^{(n)}$. Since the spectrum of any strictly cyclic operator consists entirely of compression spectrum, by Proposition $2, T M_{j} \subset$ $M_{j+1}$ for every $j$. Thus, since $f_{k} \in M_{k}^{(n)}$, we have $f_{k}^{i}=\left(T^{(n)}\right)^{j} f_{k} \in M_{k+j}$. Let

$$
N_{j}=f_{k} \vee f_{k}^{1} \vee f_{k}^{2} \vee \cdots \vee f_{k}^{j} \vee M_{k+j+1}^{(n)} \text {. }
$$

Then $N_{j}$ has finite codimension (since $M_{k+j+1}^{(n)}$ has finite codimension). Also, $N_{j} \in$ Lat $T^{(n)}$ since $M_{k+j+1}^{(n)} \in$ Lat $T^{(n)}$ and $T^{(n)} f_{k}^{i}=f_{k}^{i+1}$.

We will be done if we can show that $G=\bigcap_{j=1}^{\infty} N_{j}$. Now, since $\bigcap_{j=1}^{\infty} M_{k+j+1}^{(n)}=\{0\}$, it follows that

$$
\bigcap_{j=1}^{\infty} N_{j}=f_{k} \vee\left(\bigvee_{i=1}^{\infty} f_{k}^{i}\right) .
$$

Hence, by the definition of $f_{k}^{i}, \bigcap_{j=1}^{\infty} N_{j}$ is the smallest invariant subspace of $T^{(n)}$ containing $f_{k}$, which is exactly the definition of $G$. This completes the proof.

In the next theorem, we extend Theorem 1. It will be convenient to introduce some terminology. We define $H^{(\infty)}$ to be the collection of all square-summable sequences of vectors from $H$, and if $T \in B(H)$, we define $T^{(\infty)}$ on $H^{(\infty)}$ by $T^{(\infty)}\left(x_{1}, x_{2}, \ldots\right)=\left(T x_{1}, T x_{2}, \ldots\right)$. It is straightforward to verify that $H^{(\infty)}$ is a Hilbert space, and that $T^{(\infty)}$ is bounded. If $A$ is a subalgebra of $B(H)$, then $A^{(\infty)}$ is defined to be $\left\{T^{(\infty)}: T \in A\right\}$. If $A$ has Donoghue lattice $\left\{\{0\}, N_{n}\right\}$, if $M \in$ Lat $A^{(\infty)}$ and $M \neq\{0\}$, and if $n$ is the largest nonnegative integer such that $N_{n}$ contains every coordinate projection of $M$, then $n$ will be called the order of $M$. (Such an $n$ must exist since $N_{0}=H$.)

Theorem 3. If $A$ is an Abelian, hereditarily strictly cyclic algebra with Donoghue lattice $\left\{\{0\}, N_{n}\right\}$, then every element of Lat $A^{(\infty)}$ is a span of invariant graph subspaces.

Proof. Let $M \in$ Lat $A^{(\infty)}$. Choose a graph subspace $G_{0,1} \subset M$ as we did in the proof of Theorem 1. In that proof, we chose the domain of $G$ by taking a coordinate projection of $M$ which contained every other coordinate projection. Here we modify that procedure by insisting that the coordinate index $i_{0}$ of the domain of $G_{0,1}$ be as small as possible (there may be more than one coordinate projection of $M$ which could be chosen). As in that earlier proof, if $K_{0,1}$ is the $i_{0}$ th kernel of $M$, $M=G_{0,1} \vee k_{0,1}$. Continuing in this manner, we get a sequence of graph subspaces and a sequence of kernels such that

$$
\begin{aligned}
M & =G_{0,1} \vee K_{0,1}=G_{0,1} \vee G_{0,2} \vee K_{0,2} \\
& =G_{0,1} \vee G_{0,2} \vee \cdots \vee G_{0, i} \vee K_{0, i}
\end{aligned}
$$

If $\cap_{i=1}^{\infty} K_{0, i}=\{0\}$, we are done. If not, let $M_{1}=\bigcap_{i=1}^{\infty} K_{0, i}$. Then $M=\left(\bigvee_{i=1}^{\infty} G_{0, i}\right)$ $\vee M_{1}$.

Claim. The order of $M_{1}$ is greater than the order of $M$.

Proof of Claim. Let $r$ be the order of $M_{1}$ and $s$ the order of $M$. If $r \ngtr s$, i.e. if $r=s$, then there is an index $i$ such that the $i$ th coordinate projection of $M_{1}$ is $N_{s}$. Since $N_{s}$ contains every coordinate projection of $M$, and since we chose the domain 
for every graph by choosing the coordinate projection with lowest possible index, the $i$ th projection must have been one of the choices. But then $N_{s}$ could not be a projection of $M_{1}$. This contradiction proves the claim.

Now we proceed with $M_{1}$ as we did with $M$, getting a sequence of graphs and kernels so that

$$
M_{1}=G_{1,1} \vee K_{1,1}=G_{1,1} \vee G_{1,2} \vee \cdots \vee G_{1, i} \vee K_{1, i}
$$

If $\bigcap_{i=1}^{\infty} K_{1, i}=\{0\}$, we are done. If not, let $M_{2}=\bigcap_{i=1}^{\infty} K_{1, i}$. We continue inductively, getting a sequence of graph subspaces and a sequence $\left\{M_{i}\right\}$ of intersections of kernels, where the order of $M_{i+1}$ is grenter than the order of $M_{i}$ for all $i$. We have, for each $k$,

$$
M=\bigvee_{i=0}^{k}\left(\bigvee_{j=1}^{\infty} G_{i, j}\right) \vee M_{k}
$$

Finally, since the orders of the $M_{k}$ 's go to infinity,

$$
\bigcap_{k=1}^{\infty} M_{k}=\{0\} \text { and } M=\bigvee_{i=0}^{\infty}\left(\bigvee_{j=1}^{\infty} G_{i, j}\right) \text {, }
$$

and the proof is complete.

\section{REFERENCES}

1. W. B. Arveson, A density theorem for operator algebras, Duke Math. J. 34 (1967), 635-647.

2. W. F. Donoghue, Jr., The lattice of invariant subspaces of a completely continuous quasi-nilpotent transformation, Pacific J. Math. 7 (1957), 1031-1035.

3. M. Embry, Maximal invariant subspaces of a strictly cyclic operator algebra, Pacific J. Math. 49 (1973), 45-50.

4. C. Foias and J. P. Williams, Some remarks on the Volterra operator, Proc. Amer. Math. Soc. 71 (1972), 177-184.

5. P. R. Halmos, Finite-dimensional vector spaces, Van Nostrand, Princeton, N. J., 1958.

6. J. H. Hedlund, Strongly strictly cyclic weighted shifts, Proc. Amer. Math. Soc. 57 (1976), 119-121.

7. D. A. Herrero, Transitive operator algebras containing a subalgebra of finite strict multiplicity, Rev. Un. Mat. Argentina 26 (1972), 77-83.

8. O Operator algebras of finite strict multiplicity, Indiana Univ. Math. J. 22 (1972), 13-24.

9. D. A. Herrero and A. L. Lambert, On strictly cyclic algebras, P-algebras, and reflexive algebras, Trans. Amer. Math. Soc. 185 (1973), 229-235.

10. E. Kerlin and A. L. Lambert, Strictly cyclic shifts on 1, Acta. Sci. Math. 35 (1973), 87-94.

11. A. L. Lambert, Strictly cyclic operator algebras, Dissertation, Univ. of Michigan, 1970.

12. __ Strictly cyclic weighted shifts, Proc. Amer. Math. Soc. 29 (1971), 331-336.

13. __ Strictly cyclic operator algebras, Pacific J. Math. 39 (1971), 717-726.

14. A. L. Lambert and T. R. Turner, The double commutant of invertibly weighted shifts, Duke Math. J. 39 (1972), 385-389.

15. N. K. Nikolskii, Invariant subspaces of weighted shift operators, Math. USSR-Sb. 3 (1967), 159-176.

16. E. A. Nordgren, Closed operators commuting with a weighted shift, Proc. Amer. Math. Soc. 24 (1970), 424-428.

17. __ Invariant subspaces of a direct sum of weighted shifts, Pacific J. Math. 27 (1968), 587-598.

18. H. Radjavi and P. Rosenthal, Invariant subspaces, Springer-Verlag, Berlin, Heidelberg, New York, 1973.

19. E. Rosenthal, Power bounded strictly cyclic operators, Proc. Amer. Math. Soc. 72 (1978), 276-280. , A Jordan form for certain infinite-dimensional operators, Acta. Sci. Math. 41 (1979), $365-374$.

21. A. L. Shields, Weighted shift operators and analytic function theory, Math. Surveys, Vol. 13, Amer. Math. Soc., Providence, R.I., 1974.

Department of Mathematics, University of New Haven, West Haven, Connecticut 06516 